\section{THE APPROACHING REVISION}

oF

\section{THE BRITISH PHARMACOPEIA.}

VII.

\section{ACTIVE PRINCIPLES AND GALENICAL} PREPARATIONS.

By RALPH STOCKMAN, M.D., F.R.C.P.E.,

Lecturer on Materia Medica and Therapeutics in the School of Medicine, Edinburgh; Assistant-Physician to the Royal Infirmary.

IT has frequently been suggested that a more extended substitution of the pure active principles of plants for the Galenical preparations so generally adopted in our national Pharmacopoia would lead to much greater accuracy of dosage, and in consequence to increased precision and success in the treatment of disease. It is urged, and with great justice, that plants vary, often to a very considerable extent, in the amount of active substances which they elaborate during their growth; that this is affected not only by climate, habitat, soil, and mode of cultivation, but that even when grown in the same locality one year's crop may differ greatly from the next year's, according to the character of the season and other unavoidable circumstances.

To take two examples only, the amount of morphine in opium may vary from 4 to 15 per cent., or even more widely, while cinchona barks may yield from about 2 to 8 per cent. of quinine. Further, carelessness in drying and packing drugs may cause their active principles to decompose, as has often been the case with coca leaves, and thus the action may be greatly weakened or even entirely lost. It is well known that many good observers formerly denied that coca had any physiological effects, owing to chemical decomposition of the alkaloids having occurred in the specimens with which they experimented. Adulteration and the substitution of immature plants act in a similar way.

In the case of opium and the cinchona bark precautions have been taken in the Pharmacopoia to avoid the ill-effects of such variability in alkaloidal strength by prescribing certain kinds of opium and cinchona bark which alone are to be used in making the Galenical preparations, and also by giving exact methods for quantitative analysis; but in the majority of cases a great deal is left to chance, and no rules are laid down for the chemical examination of crude pro. ducts. In point of fact, however, the pharmacopoial authorities can scarcely be held to blame for this, as, owing to our defective knowledge of the chemistry of many of our commonest drugs, it is quite impossible to give methods for quantitative estimation of their active principles. Hence, our tinctures, extracts, and other Galenical preparations reproduce, and may even exaggerate, the original want of uniformity in the strength of the crude substances. We may even lapse further into error by trusting to our Galenical pharmacy, as in the case of jaborandi, where the leaflets contain several alkaloids, one of which. jaborine, has been shown to have an action similar to that of atropine in checking perspiration, and is, therefore, a direct antagonist to pilocarpine. By administering any of the three official Galenical preparations of the leaves instead of pilocarpine itself we run the very serious risk of producing in our patients effects exactly the opposite of those we desire. The advantages of using solutions of pure substances instead of complex pharmaceutical preparations are, therefore, extremely obvious in some cases and for some purposes; but on looking more closely into the matter it will be found that in our present Pharmacopeia this has been already recognised to a very considerable extent, and that, although some improvements and changes in this direction are possible, yet they cannot be so many nor so great as one might at the first glance conclude. The difficulties in the way depend chiefly on our defective chemical and therapeutical knowledge of drugs. In some cases we do not know what the active principles are, in others they have never been isolated pure, so that here we cannot at present improve very greatly on our pharmaceutical preparations. In other cases still there are several active substances present, and we are accustomed to rely on their combined effects. An example of this is furnished by digitalis leaves, which contain at least three active principles of different pharmacological activity, and although it would undoubtedly be an advantage to use one or more of them in a pure state and in a uniform preparation, yet nobody has ever worked up the subject clinically or chemically with such exactitude and detail that one can feel jistified in departing from the use of the time-honoured preparations of the leaves. In this, as in many other cases, the compilers of the Pharmacopoia have no proper data on which to found any reforms. As regards ergot, where reliable dosage is also very desirable, our chemical knowledge is still more deficient, and at present it would be hopeless even to attempt to make preparations of its active constituents. Hyoscyamus leaves furnish us with another example of a drug containing more than one active principle, namely, hyoscyamine and hyoscine, to the latter of which is due very largely its superior sedative properties as compared with belladonna. We have no definite knowledge, however, of the relative proportions of the two alkaloids, and hence a mixed pure solution is scarcely to be thought of unless the issue of the new edition is to be indefinitely postponed until such points can be worked up and determined.

But, further, from a therapeutical point of view it is not always preferable to use pure active principles in place of Galenical preparations. To take again the familiar remedies morphine and quinine: when the general action of these drugs is desired, it is undoubtedly most advisable to give them pure. as they are then more easily administered and more quickly absorbed, and in consequence the beneficial effect is more rapid and decided. When, however, it is desired to act on the alimentary canal, opium or its Galenical preparations are superior to morphine; the morphine is but slowly extracted from it, hence its action is more gradual and local, and is exerted on a much larger tract of the intestinal canal before complete absorption ultimately occurs. The same is true regarding quinine and the preparations of cinchona bark, the latter containing in addition tannin and other bodies, the action of which is sometimes desirable, and for this they are purposely given. In such cases the disadvantages of somewhat inaccurate dosage are more than compensated for by other advantages which the Galenical preparations possess. In the case of other large classes of drugs, such as bitters and anthelmintics, where we wish the drug to remain as long as possible in the alimentary canal, and where quick absorption is a disadvantage, we probably get much better results from the pharmaceutical preparations than we would get from active principles. Sufficient information, however, is wanting on this as on so many subjects of the same nature, for we use santonin with great advantage, and it is possible that filicic acid might prove just as good as the liquid extract of male fern. If so, its use would be a distinct improvement, as the official preparation is often weakened by chemical changes occurring in its active principle.

The numerous bodies, such as kino and catechu, which owe their activity to tannic acid, certainly act better as intestinal astringents than pure tannic acid does, and it would be no improvement to use their active principles instead of themselves. Still, there is no reason why the ointments of galls should not be made with tannic acid instead as at present, as they would then be of more definite and constant strength. Elaterium is a substance which might well be omitted from the Pharmacopoia, as its active principle, elaterin, is official, and ought always to be used in preference. Elaterin is often adulterated and of uncertain strength, and, the authorities having made a distinct improvement of this kind, should proceed to its logical conclusion, and strike out the inferior preparation.

Another active principle which is official is aloin, and it might probably with advantage, certainly with increased accuracy of dosage, replace aloes in many of our official preparations and in prescribing. In the case of nux vomica, which contains only strychnine and the much less active alkaloid brucine, which has a precisely similar action, there seems to be no good reason why any Galenical preparations at all should be retained in the Pharmacopaia. Often as the tincture and extract of nux vomica are prescribed, their 
place could be taken perfectly by strychnine or one of its salts, and there would be in consequence a great increase in accurate administration. The same is true to a very large extent of belladonna and atropine. If medical men would accustom themselves to prescribe the alkaloid instead of the tincture, extract, or succus, as the case may be, they would know exactly how much atropine the patient was getting, a matter which is left at present very much to chance. The essential oils also offer a field for many similar improvements.

It is to be confidently expected that the authorities, in preparing the new edition of the Pharmacopoesa, will keep such possible reforms in view, as the administration of reliable preparations of constant strength is admittedly all important for success in treatment.

\section{THE ANTITOXIN TREATMENT OF DIPHTHERIA.}

\section{CASES TREATED BY DR. KLEIN'S ANTITOXIN.} GREENOCK INFIRMARY.

Dr. James Wallace, Physician to the Infirmary, sends the following report of two cases :

CASE I -A. H , a girl, aged 3 , was on January 27 th transferred to the fever wards from the surgical wards of the Greenock Infirmary, where she had since October 2nd been under treatment for extensive burns of the chest, abdomen and arms. About January 24 th the patient became affected with cough. On the $27 \mathrm{th}$ the breathing became difficult, and it was then found that the pharynx and tonsils were deeply reddened and swollen, and that there was a distinct patch of greyish membrane about the size of a threepenny piece over the right tonsil. The breathing was laboured and rapid; inspirations when the child cried were of a distinctly croupy character. Both sides of the throat were swollen externally; the pulse was 132 , the temperature $101.4^{\circ}$. The bacilli of diphtheria were discovered by the Clinical Research Association in the secretions from the faures. At 6 P.M., $1 \frac{1}{2}$ drachm of Klein's antitoxin was injected between the shoulders.

January 28 th. Had passed a good night. The throat was without any sign of membrane, and the swelling was less. Breathing was calm as long as the child was not disturbed, but when she cried there was still evidence of obstruction; pulse still 132 but temperature $99.4^{\circ}$; in evening pulse 128 , temperature $101^{\circ}$.

January 29th. There was decided improvement in the child's appearance. Throat much better, cough easier, nourishment taken freely; pulse 120, temperature $99.8^{\prime \prime}$; evening pulse 112 , temperature $100.4^{\circ}$.

January 30th. Pulse 128, temperature $96.8^{\circ}$. After this the improvement was uninterrupted.

On February 3rd all swelling had disappeared; the breathring was without the slightest indication of obstruction; the urine kept free from albumen

CASE II.-C. N., a girl. aged $1 \frac{1}{2}$, was admitted on February -2nd, the disease being of four days' duration. She had a very anæmic and emaciated appearance. The breathing was rapid but not laboured and without any croupy character. The uvula, both sides of the fauces, and the back of the pharynx were swollen and covered with a dirty white membrane. The pulse was 160 , feeble, and the temperature $101^{\circ}$. Though the - case looked very unpromising, $1 \frac{1}{2}$ drachm of Klein's anti itoxin was, at 11 A.M. on February 3 rd, injected between the shoulders. At 2 P.M. the pulse was 176 , but rather firmer. The patient, however, became gradually weaker and died early next morning. Three swabs from the pharynx were examined hy the Clinical Research Association, who have sent the following report:

"Fxamination shows large numbers of bacteria, but no bacilli diphtheriæ. Culture: In twenty-four hours the medium became almost covered with masses of fungus growth, not resembling diphtheria. Microscopic examination of culture shows that the growth is composed mainly of a large jointed fungus, probably of putrefactive origin. There are also present numerous cocci arranged in pairs."
Royal Alexandra Hospital for Sick Children, Brighton.

R. Crofron-Atkins, M.B., House-Surgeon, sends the following report of a case under the care of Dr. CHAFFer :

A. W., aged 1 year and 10 months, was in good health up to January $7 \mathrm{th}$, when he was languid. On January $8 \mathrm{th}$ his breathing was noisy.

On January 9 th, at 9.30 A.M., when admitted into the hospital, he was pale and slightly cy anosed; there was much laryngeal stridor, and considerable retraction of the lower rilss. Temperature $102.9^{\circ}$, pulse 160 , respiration 52. Just above the right tonsil there was a patch of greyish-white membrane firmly adherent; the lymphatic glands were not enlarged.

3 jss of Dr. Klein's antitoxin was injected, and vin. antimoniale $m$ iij given every four hours. At 6 P.M. the temperature had risen to $103.8^{\circ}$, but the general condition remained much the same.

January 10th. Much worse. Pulse 166, extremely feeble: respirations 60 ; temperature $103^{\circ}$. There was more pallor and cyanosis, and more distress in breathing, with a greater amount of recession of the lower ribs. There was no extension of membrane. The need for operation not being very urgent, tracheotomy was postponed, and 3 jss of antitoxin was injected. After this he seemed to improve for a few hours, but towards the evening he became definitely worse, expiration being especially stridulous, and tracheotomy was performed. No membrane was seen during the operation. nor was any expectorated afterwards. The urine contained no albumen. 10.30. P.M. 3 jss of antitoxin injected.

January 11th. He had passed a fairly comfortable night; temperature $104.2^{\circ} \mathrm{F}$., pulse 142 , respirations 60 . He was flushed and sweating. The membrane had entirely disappeared from the fauces. During the day he improved con. siderably, and in the evening the temperature had fallen to $102^{\circ}$ and the pulse to 120 . From this day all the symptoms progressively improved. Dr. Klein reported that the mucus from the throat contained distinct diphtheria bacilli.

On January 20 th the temperature rose to $101^{\circ}$, and on the left side there were several patches of urticaria.

January 21st. Urticaria over both ankles, and a few more spots on the trunk. The temperature was $102^{\circ}$, but fell to normal at night. The following morning the legs, arms, and face were thickly covered witn the rash, which evidently caused him much distress. The temperature was $101^{\circ}$ and the pulse 140 , respirations 46

On January $23 \mathrm{rd}$ the eruption was fading and the temperature falling, and by the next day it had gone. No signs of paralysis showed themselves.

Remarks by Dr. Chaffey. - This case is of interest both on account of the early age of the patient and the fact that no membrane was observed subsequent to the injection of the antitoxin. The operation of tracheotomy was delayed as long as possible to enable the antitoxin to exert its full iufluence, but it was clear that the latter alone could not have saved the patient.

\section{East Suffolk Hospital. Ipswich.}

Mr. E. C. EDwards, House Surgeon. sends the following report of a case under the care of Mr. BROGDEN :

Mrs. P., aged 37 was admitted on January 16th, suffering from advanced diabetes mellitus.

On February 2nd (seventeen days after admission) she first complained of her throat having been sore for the last three or four days. The temperature since admission had ranged from $97^{\circ}$ to $98^{\circ}$. The posterior pharyngeal wall and adjacent surfaces of the tonsils were coated with white membrane. The uvala was swollen, but free from membrane; the cervical glands were not enlarged.

On February 3rd the condition of the throat was much the same. Temperature $97.6^{\circ}$. The nose was becoming "stuffed up." The patient continued to swallow solid food fairly well : she was on a strict diabetic diet, mainly consisting of meat and eggs.

On February 4th the membrane covered the uvula and anterior surface of the tonsils, and had spread forwards through the nose so as to be visible at, the anterior nares. The temperature remained subnormal $\left(97^{\circ}\right)$. No glandular enlargement.

On February 5 th the throat looked much the same. The 